\title{
Particle number control for direct simulation Monte-Carlo methodology using kernel estimates
}

Cite as: Phys. Fluids 31, 062008 (2019); https://doi.org/10.1063/1.5097902

Submitted: 29 March 2019 . Accepted: 27 May 2019 . Published Online: 14 June 2019

Hossein Gorji, Stephan Küchlin, and Patrick Jenny
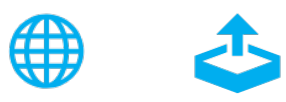

Export Citation

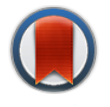

CrossMark

\section{ARTICLES YOU MAY BE INTERESTED IN}

A dusty gas model-direct simulation Monte Carlo algorithm to simulate flow in microporous media

Physics of Fluids 31, 062007 (2019); https://doi.org/10.1063/1.5094637

Controlling the bias error of Fokker-Planck methods for rarefied gas dynamics simulations

Physics of Fluids 31, 062005 (2019); https://doi.org/10.1063/1.5097884

On the concept and theory of induced drag for viscous and incompressible steady flow

Physics of Fluids 31, 065106 (2019); https://doi.org/10.1063/1.5090165

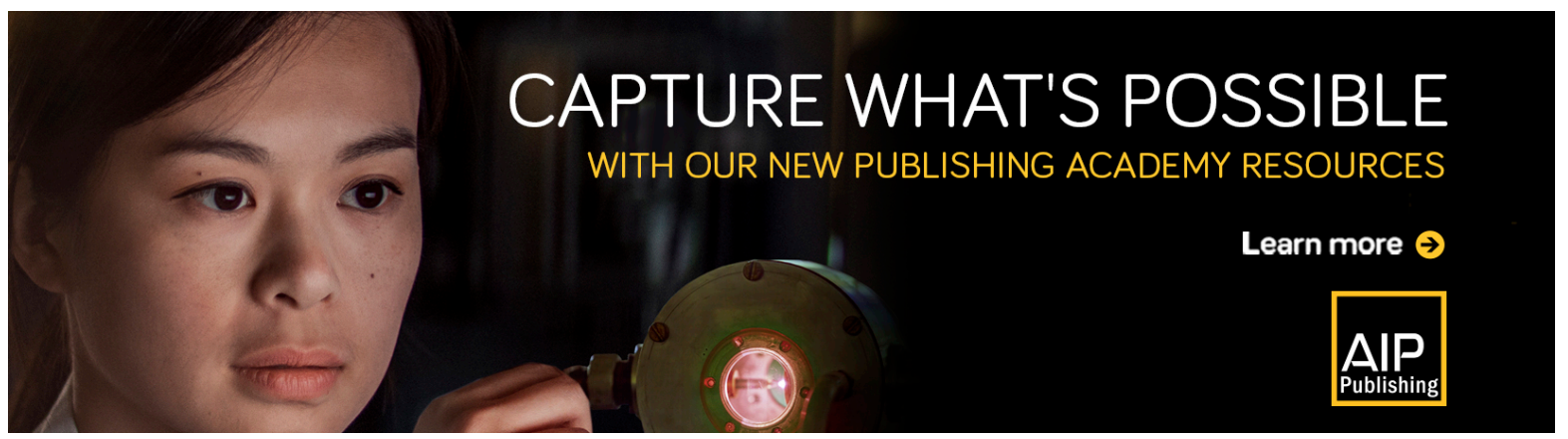




\title{
Particle number control for direct simulation Monte-Carlo methodology using kernel estimates
}

\author{
Cite as: Phys. Fluids 31, 062008 (2019); doi: 10.1063/1.5097902 \\ Submitted: 29 March 2019 - Accepted: 27 May 2019 • \\ Published Online: 14 June 2019
}

Hossein Gorji, ${ }^{1, a), b)}$ Stephan Küchlin,, ${ }^{2, a), c)}$ and Patrick Jenny ${ }^{2, d)}$

\begin{abstract}
AFFILIATIONS
${ }^{1}$ Computational Mathematics and Simulation Science, EPFL, MA C2 642, CH-1015 Lausanne, Switzerland

${ }^{2}$ Institute of Fluid Dynamics, ETH Zurich, Sonneggstrasse 3, CH-8092 Zurich, Switzerland
\end{abstract}

\author{
Note: This paper is part of the special issue on Direct Simulation Monte Carlo-The Legacy of Graeme A. Bird. \\ a) Contributions: $\mathrm{H}$. Gorji and S. Küchlin contributed equally to this work. \\ b) Electronic mail: mohammadhossein.gorji@epfl.ch \\ ${ }^{c}$ Electronic mail: kuechlin@ifd.mavt.ethz.ch \\ d)Electronic mail: jenny@ifd.mavt.ethz.ch
}

\begin{abstract}
The efficiency of stochastic particle schemes for large scale simulations relies on the ability to preserve a uniform distribution of particles in the whole physical domain. While simple particle split and merge algorithms have been considered previously, this study focuses on particle management based on a kernel density approach. The idea is to estimate the probability density of particles and subsequently draw independent samples from the estimated density. To cope with that, novel methods are devised in this work leading to efficient algorithms for density estimation and sampling. For the density inference, we devise a bandwidth with a bounded bias error. Furthermore, the sampling problem is reduced to drawing realizations from a normal distribution, augmented by stratified sampling. Thus, a convenient and efficient implementation of the proposed scheme is realized. Numerical studies using the devised method for direct simulation Monte-Carlo show encouraging performance.
\end{abstract}

Published under license by AIP Publishing. https://doi.org/10.1063/1.5097902

\section{INTRODUCTION}

The kinetic theory of rarefied gases and corresponding numerical methods comprise an essential framework to accurately account for thermo-fluid phenomena far from equilibrium. Direct Simulation Monte-Carlo (DSMC) pioneered by Bird ${ }^{1-4}$ is the most successful and widely used rarefied gas dynamics solution method, which allows for stochastic simulations of gas flows via a particle MonteCarlo algorithm. At the level of the distribution, DSMC has been shown to be consistent with solutions of the Boltzmann equation. ${ }^{5}$ Beyond that, DSMC can be employed to extract spatiotemporal autocorrelations along particle trajectories besides thermal fluctuations, both consistent with statistical thermodynamics. ${ }^{7}$ Alternative particle Monte-Carlo schemes have been introduced in order to improve the efficiency of DSMC at low Knudsen numbers ${ }^{8-12}$ or low Mach numbers. ${ }^{1}$

One of the main issues that may hinder efficient particle based simulations arises due to large variations of the particle density throughout the physical domain. The problem lies in the fact that the variance associated with estimating an observable scales inversely with the number of samples. Thus, the particle density variations can lead to poorly resolved field values and hence particle number control (pnc) measures become a crucial step toward efficient particle Monte-Carlo simulations, especially for situations where large variations of the density are expected. This issue can be encountered in various rarefied gas flow examples, including near-vacuum, hypersonic, and axisymmetric flows. ${ }^{2,15,16}$ It is important to bear in mind, however, that particle number control schemes aim at reproducing the original solution only at the (Eulerian) distribution level. Therefore, they are not relevant for situations where Lagrangian statistics (e.g., statistics along a particle trajectory) are of interest.

The issue of particle split and merge has been addressed in particle Monte-Carlo methods arising in different contexts. The most basic approach is to simply copy the particles to increase and randomly remove some to reduce their number. This naive approach 
can actually be shown to be statistically consistent, providing appropriate scaling of particle weights. This algorithm has been applied for DSMC in Ref. 15, as well as in other contexts including, e.g., turbulent flows. ${ }^{1}$

Although simple and straightforward to implement, the mentioned split and elimination algorithm is prone to numerical errors. In the split phase, the newly generated samples are not independent from the original ones. Therefore, strictly speaking, a Monte-Carlo approach should not be employed on the new samples. While over some spatiotemporal scales the newly generated samples become independent ones, the associated length and time scales may become huge and thus a constraint if, e.g., high Knudsen number flows are of interest.

An alternative particle splitting scheme can be constructed based on probability density estimators. The idea is that if the governing distribution is known or can be inferred, then new independent samples can be drawn. This idea has been employed in Ref. 18 in the context of Particle-In-Cell (PIC) methods. Yet, the mentioned study utilized a quite expensive scheme for generating new particles. Furthermore, the optimality of their considered kernel bandwidth was not taken into account, which further limits the efficiency. A particle number control scheme for the stochastic weighted particle method has been derived in Ref. 19 by introducing a weight transfer function. Kernel based density estimators have been employed for reconstructing the probability density of particles in low-variance DSMC or Fokker-Planck Monte-Carlo schemes. ${ }^{20,21}$ On the reduction side, elaborate schemes have been introduced in numerous works. ${ }^{18,22,23}$ For example, in Ref. 23, an octree structure is employed for merging nearest neighbor particles in the sample space.

This paper addresses the issue of split and merge as a sampling problem with emphasis on two aspects. First, we try to construct an accurate kernel density estimate (KDE), which can be practically applied in DSMC type simulations. Second, an efficient sampling scheme from the KDE is devised, which allows us to efficiently integrate the algorithm into existing particle Monte-Carlo codes. Once those two steps of the probability density function construction and sampling are taken, the scheme can be employed for generating new particles. Since the generated particles replace the old ones, the scheme can be used for increasing as well as reducing the number of employed particles.

Along the above-mentioned scopes, the remaining article is structured as the following. Section II addresses the estimation of an accurate probability density function from scattered data points. We rely on a KDE approach with Gaussian kernels. The reason for choosing this scheme is its physical relevance to kinetic models besides the fact that the sampling is not much involved. Section III derives the corresponding algorithms for efficient sampling from the devised kernel estimate. Validations are provided in Sec. III, and in Sec. V, the paper concludes with a summary of the highlight of this contribution and some suggestions of possible future extensions.

\section{REVIEW OF KERNEL DENSITY ESTIMATION}

Density estimation is the problem of finding the underlying probability density from a set of scattered data points. Two nonparametric density estimation approaches rely on histograms and kernels. Typically, kernel estimates provide a faster convergence rate. ${ }^{24}$ Also, as shown later, the sampling problem is quite straightforward. Therefore, we focus on the KDE approach in the following.

\section{A. Univariate distributions}

Suppose a set of $N$ particles with velocities $\left\{M^{(i)}\right\}_{i=1}^{N}$ is given. In the univariate density estimation, we wish to infer the probability density $f(v)$, where $v$ is a scalar. Hence, the set $\left\{M^{(i)}\right\}_{i=1}^{N}$ is comprised of $N$ independent realizations in the probability space over which $f(v)$ is defined. There exist different approaches for estimating $f($.$) . In the KDE approach, { }^{25,26}$ the ansatz

$$
\hat{f}_{N, h}(v)=\frac{1}{N h} \sum_{i=1}^{N} K\left(\frac{v-M^{(i)}}{h}\right)
$$

is considered, where $h$ is the smoothing parameter. It turns out that the accuracy of $\hat{f}_{N, h}$ as an approximation of $f$ crucially depends on $h$ rather than on the choice of $K($.). For convenience of generating new realizations from $\hat{f}_{N, h}$, we choose

$$
K(v)=\frac{1}{\sqrt{2 \pi}} e^{-v^{2} / 2}
$$

to be the (univariate) Gaussian kernel.

A common approach to compute $h$ is to minimize the expected $L_{2}$ distance between $\hat{f}_{N, h}$ and $f$. Consider the mean integrated squared error

$$
\text { MISE }=\mathbb{E}\left(\int_{\mathbb{R}}\left(f(v)-\hat{f}_{N, h}(v)\right)^{2} d v\right) .
$$

By minimizing MISE, ${ }^{27,28}$ we obtain the optimal $h$ as

$$
h_{\text {opt }}=\frac{R(K)^{1 / 5}}{m_{2}(K)^{2 / 5} R\left(f^{\prime \prime}\right)^{1 / 5} N^{1 / 5}}
$$

in the asymptotic limit, where

$$
R(K)=\int_{\mathbb{R}} K^{2}(v) d v
$$

and

$$
m_{2}(K)=\int_{\mathbb{R}} v^{2} K(v) d v .
$$

Note that $f^{\prime \prime}$ is the second derivative of $f$. The terms $R(K)$ and $m_{2}(K)$ can be computed easily for $K$ given by Eq. (2). They read $R(K)=1 /(2 \sqrt{\pi})$ and $m_{2}(K)=1$. Since $f$ is unknown, the bandwidth Eq. (4) is not closed. However, one can consider, e.g., a truncated orthogonal expansion of $f^{\prime \prime}$ in order to close the $h_{\text {opt }}$ formula (4). There exists an immense amount of work on the KDE approach, where its accuracy and convergence are established for a large class of probability densities (see the review ${ }^{29}$ ).

The physical intuition behind this approach becomes clear by considering that new samples from a KDE approximation derive their states from original samples subject to a Gaussian noise. The introduced noise which reflects the uncertainty of original samples results in a smoother probability density estimate compared to the histogram approach. 
While $h_{\text {opt }}$ provides an optimal bandwidth in terms of MISE, its application for a particle number control routine may not be beneficial. Note that $h_{o p t}$ provides a trade-off between the bias $\operatorname{Bias}_{\hat{f}_{N, h}}$ and the variance $\operatorname{Var}_{\hat{f}_{N, h}}$ of the estimator by minimizing MISE $=\operatorname{Bias}_{\hat{f}_{N, h}}^{2}+\operatorname{Var}_{\hat{f}_{N, h}}$. To illustrate this point, consider the asymptotic case of $h \rightarrow 0$, in which the estimator collapses to the empirical density

$$
f_{e m p}=\frac{1}{N} \sum_{i=1}^{N} \delta\left(v-M^{(i)}\right),
$$

where $\delta($.$) is the Dirac delta. Here, since \mathbb{E}\left[f_{\text {emp }}\right]=f$, the bias error vanishes. However, the variance is infinitely large since

$$
\mathbb{E}\left(f_{\text {emp }}^{2}\right) \rightarrow \infty
$$

for finite $N$. In the other words, the noise in samples drawn from $f_{e m p}$ is maximal since there is no smoothing effect present in the estimator. On the other hand, $\hat{f}_{N, h}$ has a bias error of $\mathcal{O}\left(h^{2}\right)$, while the variance is of $\mathcal{O}\left(h^{-1}\right)$ for a nonvanishing $h$. $^{30}$

In order to come up with a bias-variance trade-off suitable for DSMC type simulations, we consider the bias in second order moments (proportional to temperature). According to asymptotic analysis, every choice of $h$ proportional to $N^{-1 / 5}$ recovers the correct density as $N \rightarrow \infty$. Hence, we make the ansatz

$$
h \propto \frac{1}{N^{1 / 5}}
$$

for the bandwidth. Furthermore, note that

$$
\int_{\mathbb{R}} \hat{f}_{N, h} v^{2} d v=\int_{\mathbb{R}} f v^{2} d v+h^{2} .
$$

Now, let us choose an upper bound for the error in the second moment to be a fraction of thermal energy. Thus, we require that

$$
h^{2} \leq \epsilon^{2} \frac{k T}{m}
$$

with small $\epsilon$, e.g., $\epsilon=0.01$ as chosen in this study. Here, $k$ is the Boltzmann constant, $T$ is the temperature, and $m$ is the molecular mass. Therefore, to minimize the variance and to honor the inequality (11), a closure for $h$ is made as a function of $\epsilon, N$, and thermal speed,

$$
h=\frac{\epsilon}{N^{1 / 5}} \sqrt{\frac{k T}{m}} .
$$

By inserting Eq. (12) into Eq. (10), we get a criterion to choose $\epsilon$ based on the normalized error in thermal energy $N^{-2 / 5} \epsilon^{2}$ (in the onedimensional setting). In practice, provided an error tolerance, $\epsilon$ can be then computed for a given number of particles per cell (ppc). For example, $\epsilon=0.01$ gives the thermal energy relative error of $10^{-4}$ for 100 particles per cell.

\section{B. Multivariate distributions}

More relevant for gas kinetic simulations are multivariate density estimations. The previous ansatz can easily be generalized to

$$
\hat{f}_{N, \boldsymbol{H}}(\boldsymbol{v})=\frac{1}{N}|\boldsymbol{H}|^{-1} \sum_{i=1}^{N} K\left(\boldsymbol{H}^{-1}\left(\boldsymbol{v}-\boldsymbol{M}^{(i)}\right)\right),
$$

where $\boldsymbol{H}$ is a positive definite bandwidth characterizing both size and orientation of the kernel. Note that for any $d \times d$ positive-definite $\boldsymbol{H}$ that scales with $N^{-1 /(d+4)}$, the correct density is recovered as $N \rightarrow \infty$. Following the same analysis as in the univariate case, we use the ansatz

$$
H_{i j}=\frac{\epsilon}{N^{1 / 7}} \sqrt{\frac{k T}{m}} \delta_{i j} .
$$

Even though the discussion here is focused only on monatomics, generalization of the proposed approach to polyatomic molecules with continuous degrees of freedom is possible. The sample space of the KDE probability density should be augmented to include all internal degrees of freedom of the considered species. For example, considering a diatomic molecule without active vibrational degrees of freedom, the sample space of $\hat{f}_{N, \boldsymbol{H}}$ becomes five dimensional, i.e., three velocities and two rotational degrees of freedom.

\section{PARTICLE MANAGEMENT}

In a typical particle Monte-Carlo scheme, the physical domain $\Omega \subset \mathbb{R}^{3}$ is discretized into a set of $N_{c}$ computational cells $\delta \Omega_{j}$ such that $\Omega=\cup_{j=1}^{N_{c}} \delta \Omega_{j}$. Furthermore, each particle $i$ has a weight $w^{(i)}$, velocity $\boldsymbol{M}^{(i)}$, and position $\boldsymbol{X}^{(i)}$. Their states determine the probability density through

$$
f(\boldsymbol{x}, \boldsymbol{v}, t)=\frac{1}{|\Omega| \rho(\boldsymbol{x}, t)} \sum_{i=1}^{\infty} w^{(i)} \delta\left(\boldsymbol{v}-\boldsymbol{M}^{(i)}\right) \delta\left(\boldsymbol{x}-\boldsymbol{X}^{(i)}\right),
$$

where $\rho$ is the gas density. By assuming that the probability density is uniform in each cell and by using a finite number of particles, the particle representation provides the approximation

$$
\tilde{f}(\boldsymbol{v}, t \mid \boldsymbol{x})=\frac{1}{\left|\delta \Omega_{j}\right| \rho(\boldsymbol{x}, t)} \sum_{i=1}^{N^{\left(\alpha_{j}\right)}} w^{(i)} \delta\left(\boldsymbol{v}-\boldsymbol{M}^{(i)}\right)
$$

of $f$ for $\forall \boldsymbol{x} \in \delta \Omega_{j}$. Here, the index set $\alpha_{j}$ denotes the set of particle indices whose positions belong to the cell $\delta \Omega_{j}$, and $N^{\left(\alpha_{j}\right)}$ is the number of these particles. Furthermore, for simplicity of the derivations, let here and henceforth $\left\{1, \ldots, N^{\left(\alpha_{j}\right)}\right\}$ be the set of particle indices that are in the cell $j$. Therefore, the total mass in the cell reads $\mathcal{W}_{j}=\sum_{i=1}^{N^{\left(\alpha_{j}\right)}} w^{(i)}$.

The idea of particle management is to adjust the number of particles in each cell such that better statistics can be obtained from Monte-Carlo simulations. Therefore, particle weights have to vary in space and time. In order to keep the methodology applicable for conventional DSMC, we devise the algorithm such that all particles in each cell have the same particle weight. Therefore, we require that $\forall i \in \alpha_{j}: w^{(i)}=w_{j}$, where $w_{j}$ with $j \in\left\{1, \ldots, N_{c}\right\}$ is the input. Based on mass conservation, this results in the required number of samples, i.e., $N_{j}=\mathcal{W}_{j} / w_{j}$.

Once the particles move for a time step, particles with different weights may cross boundaries of their cells. Therefore, we end up with a situation where we have particles of different weights in a computational cell $j$. The particle management algorithm presented in Secs. III A and III B introduces efficient sampling schemes based on uniform/nonuniform weight KDEs. 
ALGORITHM 1. Sampling from $\hat{f}_{N, H}$ assuming identical weights.

\begin{tabular}{l}
\hline 1: for $l \in\left\{1, \ldots, N_{j}\right\}$ do \\
2: $\quad$ Draw $i \sim \mathcal{U}\left\{1, N_{j}\right\}$ \\
3: $\quad \operatorname{Draw} \boldsymbol{M}_{s}^{(l)} \sim \mathcal{N}\left(\boldsymbol{M}^{(i)}, \boldsymbol{H}\right)$ \\
4: $\quad \boldsymbol{X}_{s}^{(l)} \leftarrow \boldsymbol{X}^{(i)}$ \\
5: end for \\
\hline
\end{tabular}

\section{A. Sampling from uniform weight KDE}

One of the main advantages of a kernel estimate of the type given by Eq. (13) with the Gaussian kernel is that sampling from the estimate does not require an acceptance-rejection method. Suppose we have a density $\hat{f}_{N, \boldsymbol{H}}$ given by Eq. (13), and let $\boldsymbol{M}_{s}$ denote the velocity of a newly generated particle, and $\boldsymbol{X}_{s}$ is the corresponding position.

If all original particles have similar weights, then generating new samples from $\hat{f}_{N, \boldsymbol{H}}$ is straightforward. Algorithm 1 provides $N_{j}$ samples.

Here, $\mathcal{U}\{a, b\}$ is the discrete uniform density between $a$ and $b$. Furthermore, $\mathcal{N}(a, b)$ denotes the normal distribution with mean $a$ and variance $b$.

\section{B. Sampling from non-uniform weight KDE}

Since we are interested in cases where particles ending up in a cell may have different weights, we need to account for different weights. In fact, the data of particles with larger weights should have a stronger influence on the samples. To formulate that, instead from $\hat{f}_{N, \boldsymbol{H}}$ given by Eq. (13), we need to sample from

$$
\hat{f}_{N, \boldsymbol{H}}(\boldsymbol{v})=\frac{1}{\mathcal{W}_{j}}|\boldsymbol{H}|^{-1} \sum_{i=1}^{N^{\left(\alpha_{j}\right)}} w^{(i)} K\left(\boldsymbol{H}^{-1}\left(\boldsymbol{v}-\boldsymbol{M}^{(i)}\right)\right) .
$$

Intuitively, first, we need to assign to each particle $i$ a partition of $[0,1]$, proportional to its weight. Then, we determine the interval where a uniformly distributed random number between 0 and 1 falls in. Once we find that interval, we can simply draw a normally distributed random number with mean being the velocity of the particle whose interval has been chosen and the covariance $\boldsymbol{H}$. An efficient formulation of this idea is known as stratified sampling, which is reviewed in the following. ${ }^{31}$ Let us define the partitions by setting $\hat{S}_{0}=0$ and

$$
\hat{S}_{k}=\mathcal{W}_{j}^{-1} \sum_{i=1}^{k} w^{(i)}
$$

with corresponding intervals

$$
\mathcal{I}_{k}=\left[\hat{S}_{k-1}, \hat{S}_{k}\right]
$$

for $k \in\left\{1, \ldots, N^{\left(\alpha_{j}\right)}\right\}$. The idea is then to generate a set of ordered uniformly distributed random numbers to choose from which interval we should sample. Algorithm 2 shows how to generate $N_{j}$ particles from $\hat{f}_{N, \boldsymbol{H}}$ [Eq. (17)].
ALGORITHM 2. Sampling from $\hat{f}_{N, H}$ composed of different weights.

$$
\begin{array}{ll}
\text { 1: } & \text { for } l \in\left\{1, \ldots, N_{j}\right\} \text { do } \\
\text { 2: } \quad \text { Draw } R_{f} \sim \mathcal{U}[0,1] \\
\text { 3: } \quad R_{f} \leftarrow\left((l-1)+R_{f}\right) / N_{j} \\
\text { 4: } \quad \text { Find } k \text { such that } R_{f} \in \mathcal{I}_{k} \\
\text { 5: } \quad \text { Draw } \boldsymbol{M}_{s}^{(i)} \sim \mathcal{N}\left(M^{(k)}, H\right) \\
\text { 6: } & \text { end for }
\end{array}
$$

Here, $\mathcal{U}[a, b]$ is the continuous counterpart of $\mathcal{U}\{a, b\}$. The fact that there is no need to employ an acceptance-rejection algorithm makes Algorithm 2 very attractive.

\section{Summary}

In this subsection, we provide a brief summary of the particle management algorithm. Suppose the physical domain is discretized into $N_{c}$ computational cells and that weights $w_{j}$ are provided for $j \in\left\{1, \ldots, N_{c}\right\}$. Initially, each cell $j$ gets populated by $N^{\left(\alpha_{j}\right)}$ with total mass $\mathcal{W}_{j}=\sum_{i \in \alpha_{j}} w^{(i)}$. The following summary describes each step of the algorithm for a given time step.

1. Move particles in the physical space.

2. Apply the boundary conditions.

3. For each cell $j$

(a) Compute the target number of particles $N_{j}=\mathcal{W}_{j} / w_{j}$.

(b) If $N_{j}=N^{\left(\alpha_{j}\right)}$ and if particles have similar weights, go to $3 \mathrm{f}$.

(c) Compute the bandwidth from Eqs. (12) and (14).

(d) If the particles have similar weights, run Algorithm 1, otherwise Algorithm 2 to generate $N_{j}$ new particles.

(e) Remove the original particles.

(f) Update particle velocities according to the governing equations.

4. Estimate the observables.

Note that treatment of boundary conditions remains identical without/with the particle number control scheme. This is because the devised approach does not alter the governing equation, yet it targets an improved particle distribution in the physical domain.

\section{SIMULATION STUDIES}

While improvements resulting from particle number control schemes can be highlighted in scenarios with sharp density variations, in the following simulation study, we pursue another objective. In particular, we evaluate the proposed algorithm by achieving the target of a uniformly distributed set of particles while the observables remain unbiased. Even though the results discussed here serve as a preliminary evaluation of the algorithm, we anticipate that more challenging test cases (omitted due to brevity) can be treated by the introduced scheme in a straightforward manner. Nevertheless, we postpone those more demanding simulation settings to future studies.

The test case is a two-dimensional supersonic flow around an inclined plate as studied in Ref. 2. Flow of argon at Mach 5 is considered around a flat plate with an incidence angle of $30^{\circ}$. 


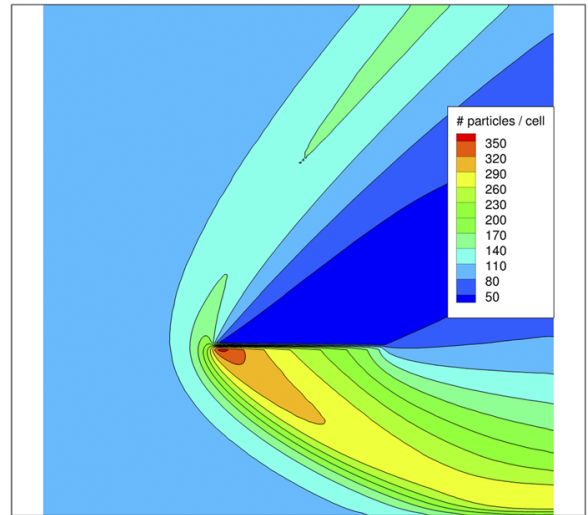

(a)

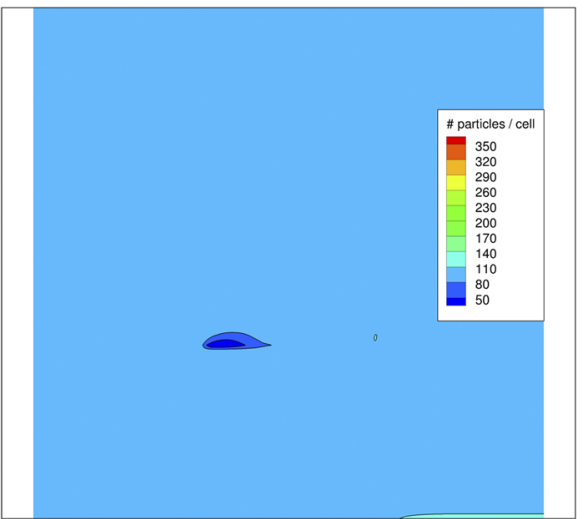

(b)
The mean-free-path at inflow is $9.25 \mathrm{~mm}$, and thus, a uniform mesh of $200 \times 200$ cells is chosen resulting in a cell spacing of $3.23 \mathrm{~mm}$, which is well below the mean free path. Similarly, the time step size is set to $1.05 \mu \mathrm{s}$ in order to capture the mean collision time.
The standard no-time-counter algorithm of Bird for variable hard sphere molecules is employed, and the surface is modeled as a fully diffusive wall. The results are sampled after 6000 initial time steps.
FIG. 1. Particle number distribution for the case with $N_{p}=100$ (a) without particle number control and (b) with particle number control.

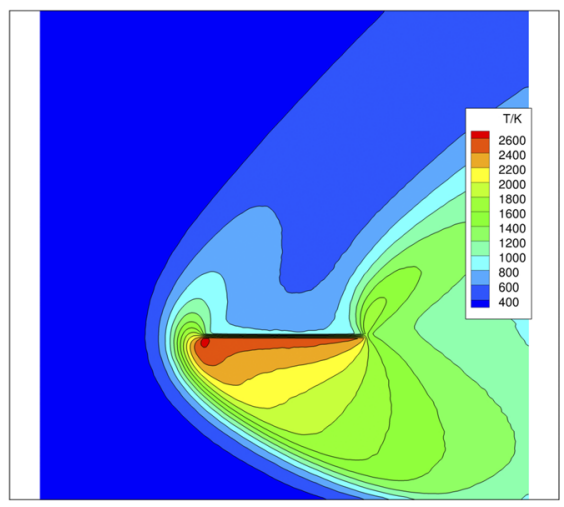

(a)

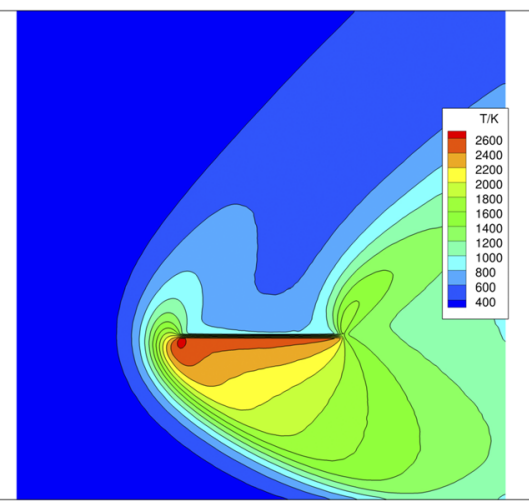

(b)

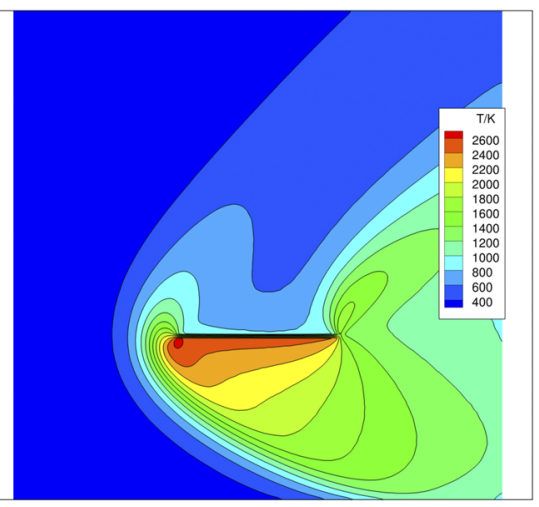

(c)

FIG. 2. Temperature contours without particle number control: (a) $N_{p}=20$, (b) $N_{p}=100$, and (c) $N_{p}=500$.

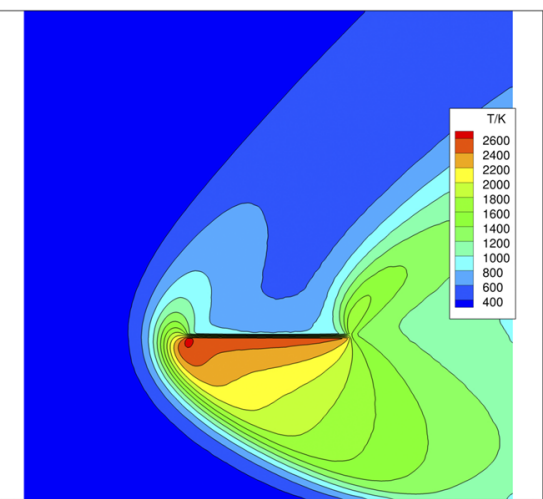

(a)

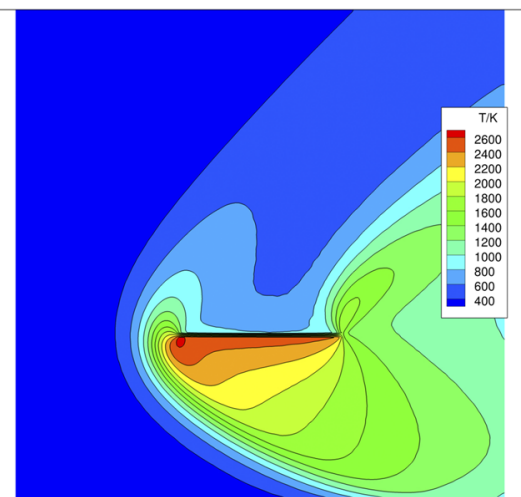

(b)

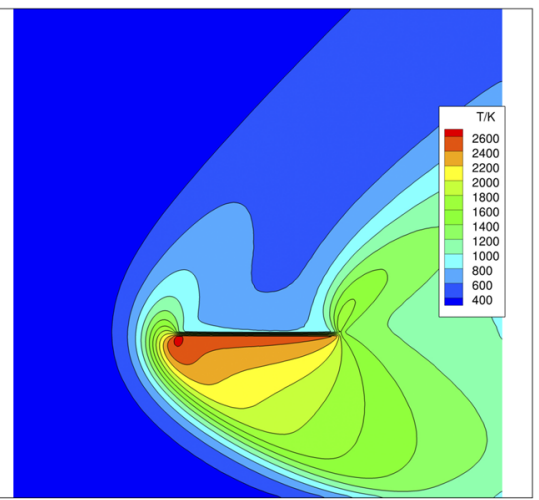

(c)

FIG. 3. Temperature contours with particle number control: (a) $N_{p}=20$, (b) $N_{p}=100$, and (c) $N_{p}=500$. 


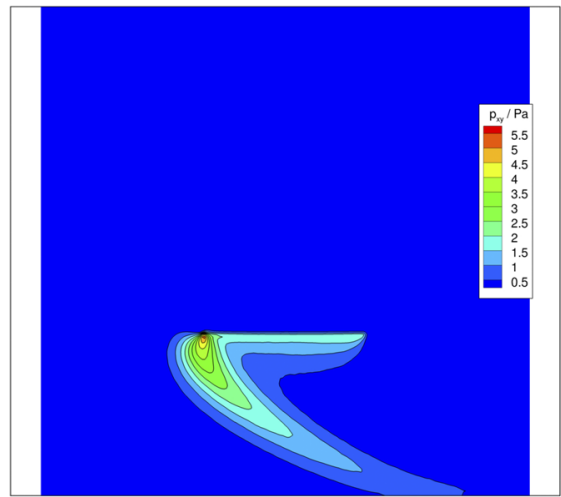

(a)

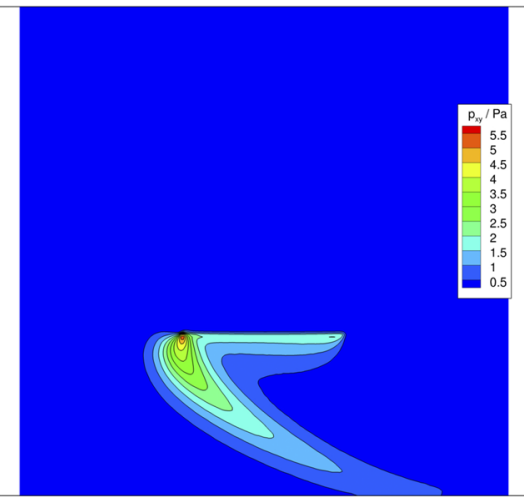

(b)

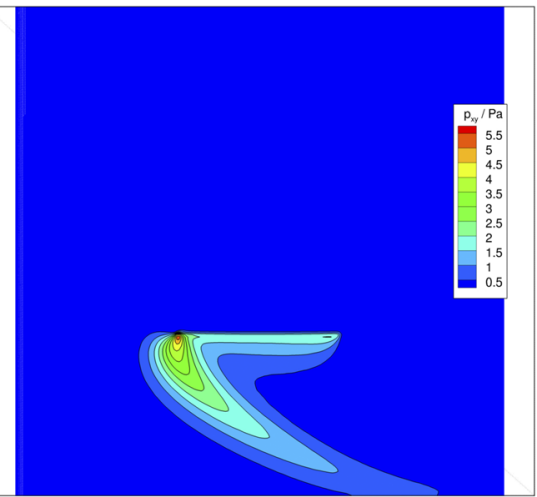

(c)

FIG. 4. Shear stress contours without particle number control: (a) $N_{p}=20$, (b) $N_{p}=100$, and (c) $N_{p}=500$.

Three different initial numbers $N_{p} \in\{20,100,500\}$ of particles per cell were considered. For each case, two simulations were conducted, i.e., one without particle number control and one with the algorithm summarized in Sec. III C. The particle number control algorithm gets activated once the number of particles per cell deviates by more than $5 \%$.

The particle number distribution in the statistically stationary state for the case with $N_{p}=100$ is shown in Figs. 1(a) and 1 (b) without and with particle number control, respectively. It can be observed that the algorithm achieves its target of maintaining a uniform particle distribution in the physical domain. The total numbers of particles at statistically stationary state read 4580000 and 3929000 without and with particle number control, respectively. Therefore, we observe roughly a $13 \%$ reduction of the total number of particles due to the devised algorithm. Consequently, a speedup of around 15\% in the run-time is achieved by applying the particle number control scheme.
The corresponding results for the temperature are shown in Figs. 2 and 3 without and with particle number control, respectively.

Very close agreements between the converged results can be observed in the upstream, shock, and downstream regions. This further confirms that the bias introduced through Eq. (12) is negligible.

Furthermore, the shear stress is shown in Figs. 4 and 5 without and with particle number control, respectively. Similar to the temperature, close agreement between these two sets of results is observed.

A more detailed quantitative comparison is performed by extracting the flow Mach number at a distance of two mean-freepaths upstream of the leading edge. The results shown in Fig. 6 depict close agreements between simulations without and with particle number control. Finally, the pressure profile over the plate shown in Fig. 7 further ensures the accuracy of the particle number control scheme.

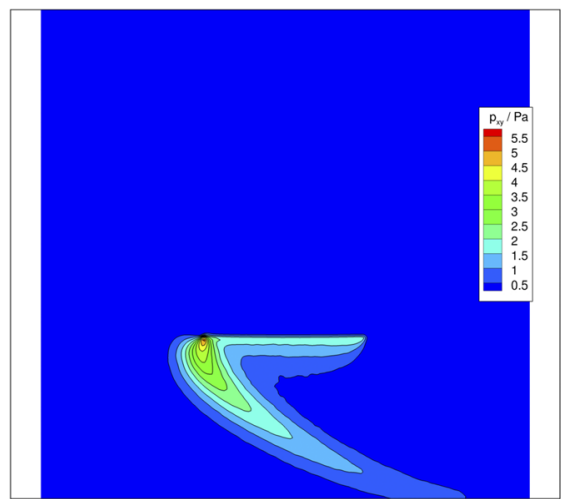

(a)

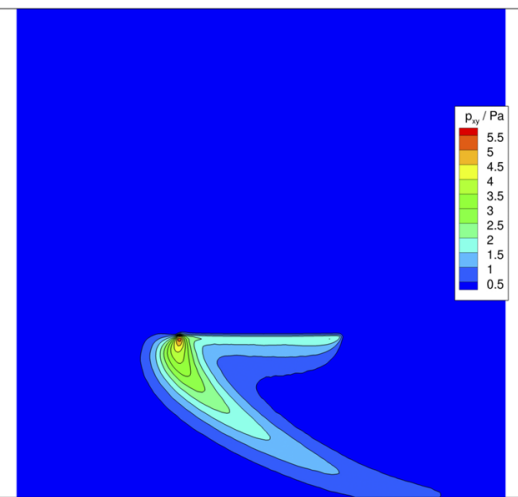

(b)

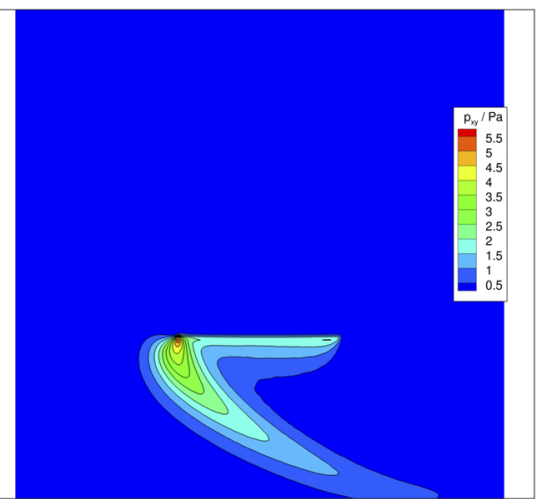

(c)

FIG. 5. Shear stress contours with particle number control: (a) $N_{p}=20$, (b) $N_{p}=100$, and (c) $N_{p}=500$. 


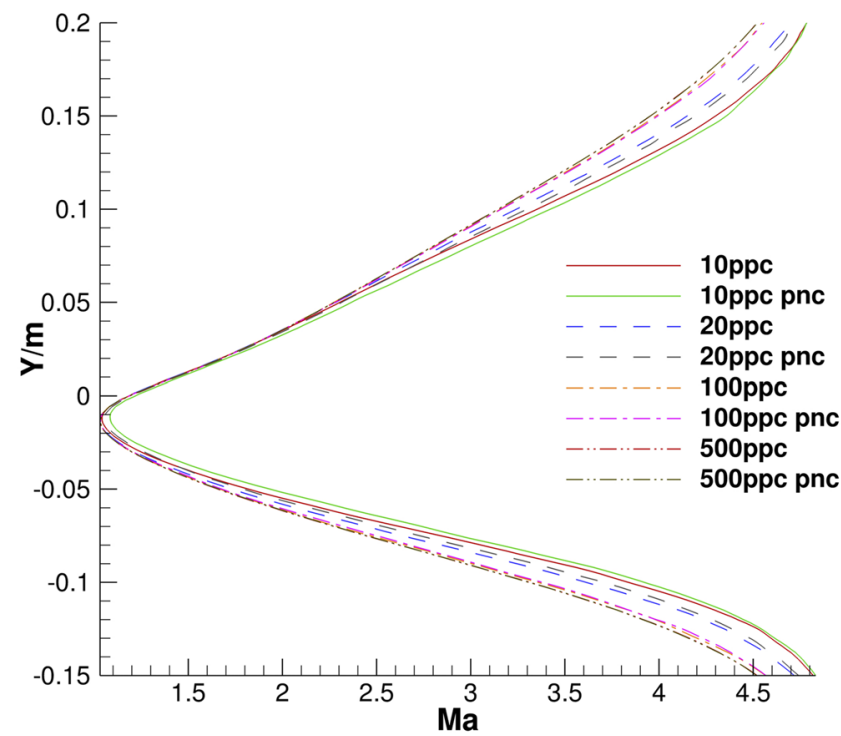

FIG. 6. Flow Mach number at two mean-free-paths upstream of the leading edge for different numbers of particles per cell $(\mathrm{ppc})$ without and with particle number control (pnc).

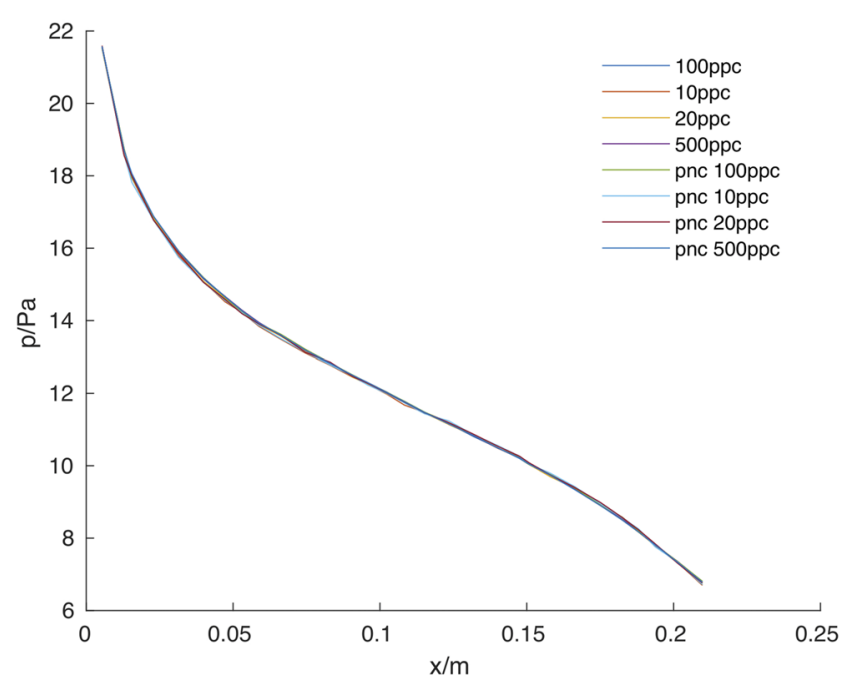

FIG. 7. Pressure distribution over the plate for different numbers of particles per cell (ppc) without and with particle number control (pnc).

\section{CONCLUSION}

In contrast to deterministic solvers, many aspects of particle Monte-Carlo methods have not been fully explored so far. This encourages further developments of methodologies dealing with different characteristics of an optimal particle Monte-Carlo algorithm. This study tackles the issue of particle number control for DSMC, with an emphasis on the kernel based bootstrapping. The issue of resampling from an estimated kernel was analyzed in terms of both bias and variance. Furthermore, an efficient sampling technique was introduced which does not rely on the acceptance-rejection scheme. A straightforward simulation study was presented for the supersonic flow around an inclined flat plate. The results obtained from DSMC with the particle number control scheme turned out to be consistent with the original DSMC method, while at the same time, the former succeeded in maintaining a uniform distribution of particles in the solution domain, resulting in roughly $15 \%$ speedup in computational time. This is encouraging since the extra cost per particle of the introduced algorithm is negligible. Future studies focus on assessment of the devised particle number control scheme for flows with sharper density gradients. Also, generalizations of the $\mathrm{KDE}$ approach for complex gas flows are pursued.

\section{ACKNOWLEDGMENTS}

Hossein Gorji acknowledges the funding provided by the Swiss National Science Foundation under Grant No. 174060.

\section{REFERENCES}

${ }^{1}$ G. A. Bird, "Breakdown of translational and rotational equilibrium in gaseous expansions," AIAA J. 8(11), 1998-2003 (1970).

${ }^{2}$ G. A. Bird, Molecular Gas Dynamics and the Direct Simulation of Gas Flows (Oxford University Press, New York, 1994).

${ }^{3}$ M. A. Gallis, J. R. Torczynski, S. J. Plimpton, D. J. Rader, and T. Koehler, "Direct simulation Monte Carlo: The quest for speed," AIP Conf. Proc. 1628, 27-36 (2014).

${ }^{4} \mathrm{~S}$. Gimelshein and I. Wysong, "DSMC modeling of flows with recombination reactions," Phys. Fluids 29(6), 067106 (2017).

${ }^{5} \mathrm{~K}$. Nanby, "Theoretical basis of the direct simulation Monte Carlo method," in Proc. Int. Symp. Rarefied Gas Dynamics, edited by V. Boffi and C. Cercignani, Stuttgart, Germany, 1986, Vol. 1, pp. 369-383.

${ }^{6}$ W. Wagner, "A convergence proof for Bird's direct simulation Monte Carlo method for the Boltzmann equation," J. Stat. Phys. 66(3-4), 1011-1044 (1992).

${ }^{7}$ A. L. Garcia, "Hydrodynamic fluctuations and the direct simulation Monte Carlo method," in Microscopic Simulations of Complex Flows (Springer, 1990), pp. 177-188.

${ }^{8}$ M. H. Gorji, M. Torrilhon, and P. Jenny, "Fokker-Planck model for computational studies of monatomic rarefied gas flows," J. Fluid Mech. 680, 574-601 (2011).

${ }^{9} \mathrm{P}$. Jenny, M. Torrilhon, and S. Heinz, "A solution algorithm for the fluid dynamic equations based on a stochastic model for molecular motion," J. Comput. Phys. 229(4), 1077-1098 (2010).

${ }^{10} \mathrm{M}$. H. Gorji and P. Jenny, "Fokker-Planck-DSMC algorithm for simulations of rarefied gas flows," J. Comput. Phys. 287, 110-129 (2015).

${ }^{11}$ S. Küchlin and P. Jenny, "Parallel Fokker-Planck-DSMC algorithm for rarefied gas flow simulation in complex domains at all Knudsen numbers," J. Comput. Phys. 328, 258-277 (2017).

${ }^{12}$ M. Sadr and M. H. Gorji, "A continuous stochastic model for non-equilibrium dense gases," Phys. Fluids 29(12), 122007 (2017).

${ }^{13}$ L. L. Baker and N. G. Hadjiconstantinou, "Variance reduction for Monte Carlo solutions of the Boltzmann equation," Phys. Fluids 17(5), 051703 (2005).

${ }^{14}$ T. M. M. Homolle and N. G. Hadjiconstantinou, "A low-variance deviational simulation Monte Carlo for the Boltzmann equation," J. Comput. Phys. 226(2), 2341-2358 (2007).

${ }^{15}$ K. C. Kannenberg and I. D. Boyd, "Three-dimensional Monte Carlo simulations of plume impingement," J. Thermophys. Heat Transfer 13(2), 226-235 (1999).

${ }^{16}$ C. Galitzine and I. D. Boyd, "Development of an adaptive weighting scheme for DSMC and its application to an axisymmetric jet," AIP Conf. Proc. 1501, 587-594 (2012). 
${ }^{17}$ M. Muradoglu and S. B. Pope, "Local time-stepping algorithm for solving probability density function turbulence model equations," AIAA J. 40(9), 1755-1763 (2002).

${ }^{18}$ M. Pfeiffer, A. Mirza, C.-D. Munz, and S. Fasoulas, "Two statistical particle split and merge methods for particle-in-cell codes," Comput. Phys. Commun. 191, 9-24 (2015)

${ }^{19}$ S. Rjasanow and W. Wagner, Stochastic Numerics for the Boltzmann Equation (Springer, 2005).

${ }^{20}$ H. A. Al-Mohssen and N. G. Hadjiconstantinou, "Low-variance direct Monte Carlo simulations using importance weights," ESAIM: Math. Modell. Numer. Anal. 44(5), 1069-1083 (2010)

${ }^{21}$ B. S. Collyer, C. Connaughton, and D. A. Lockerby, "Importance sampling variance reduction for the Fokker-Planck rarefied gas particle method," J. Comput. Phys. 325, 116-128 (2016).

${ }^{22}$ G. Lapenta and J. U. Brackbill, "Dynamic and selective control of the number of particles in kinetic plasma simulations," J. Comput. Phys. 115(1), 213-227 (1994).

${ }^{23}$ R. S. Martin and J.-L. Cambier, "Octree particle management for DSMC and PIC simulations,” J. Comput. Phys. 327, 943-966 (2016).
${ }^{24}$ L. Wasserman, All of Nonparametric Statistics (Springer Science \& Business Media, 2006)

${ }^{25}$ E. Parzen, "On estimation of a probability density function and mode," Ann. Math. Stat. 33(3), 1065-1076 (1962).

${ }^{26}$ V. A. Epanechnikov, "Non-parametric estimation of a multivariate probability density," Theory Probab. Its Appl. 14(1), 153-158 (1969).

${ }^{27}$ Z. I. Botev, J. F. Grotowski, D. P. Kroese et al., "Kernel density estimation via diffusion,” Ann. Stat. 38(5), 2916-2957 (2010).

${ }^{28}$ B. U. Park and J. S. Marron, "Comparison of data-driven bandwidth selectors," J. Am. Stat. Assoc. 85(409), 66-72 (1990).

${ }^{29}$ N.-B. Heidenreich, A. Schindler, and S. Sperlich, "Bandwidth selection for kernel density estimation: A review of fully automatic selectors," AStA Adv. Stat. Anal. 97(4), 403-433 (2013).

${ }^{30}$ D. Ruppert and D. B. H. Cline, "Bias reduction in kernel density estimation by smoothed empirical transformations," Ann. Stat. 22, 185-210 (1994).

${ }^{31} \mathrm{~J}$. D. Hol, T. B. Schon, and F. Gustafsson, "On resampling algorithms for particle filters," in 2006 IEEE Nonlinear Statistical Signal Processing Workshop (IEEE, 2006), pp. 79-82. 